\title{
LORNOXICAM-LOADED NANOSPONGES FOR CONTROLLED ANTI-INFLAMMATORY EFFECT: IN VITRO/IN VIVO ASSESSMENT
}

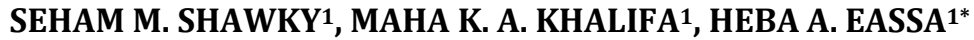

1Department of Pharmaceutics and Industrial Pharmacy, Faculty of Pharmacy, Al-Azhar University, Nasr City, Cairo 11651, Egypt Email: hebaabdullah@azhar.edu.eg

Received: 18 Aug 2020, Revised and Accepted: 01 Oct 2020

\begin{abstract}
Objective: To design a controlled topical delivery system of lornoxicam (LX) in order to enhance skin permeation and treatment efficacy. Nanosponges were selected as a novel carrier for this purpose.

Methods: Nanosponges were formulated via the emulsion solvent evaporation method using ethyl cellulose (polymer) and polyvinyl alcohol (surfactant). Nanosponge dispersions were characterized for colloidal properties, entrapment efficiency and in vitro release study. The nanosponge formulation (LS1) was then incorporated into carboxymethyl cellulose sodium hydrogels and evaluated for pH, viscosity and in vitro drug release. Skin irritation was evaluated, and anti-inflammatory activity was assessed via rat hind paw edema method.

Results: Nanosponges were in the nano-sized range and attained a uniform round shape with a spongy structure. LS1exhibited the highest LX release after $6 \mathrm{~h}$, so it was incorporated as hydrogel. Formulated hydrogels showed acceptable physicochemical parameters (pH, drug content and rheological properties). Skin irritation testing proved LX-loaded nanosponge hydrogel formulation (G1) to be non-irritant. In vivo study revealed an enhanced anti-inflammatory activity of G1 for $6 \mathrm{~h}(\mathrm{p}<0.001)$.
\end{abstract}

Conclusion: The developed nanosponge hydrogel is an efficient nanocarrier for improved and controlled topical delivery of LX.

Keywords: Ethylcellulose, Lornoxicam, Nanosponge, Topical delivery, Anti-inflammatory activity

(C) 2020 The Authors. Published by Innovare Academic Sciences Pvt Ltd. This is an open access article under the CC BY license (http://creativecommons.org/licenses/by/4.0/) DOI: http://dx.doi.org/10.22159/ijap.2020v12i6.39430. Journal homepage: https://innovareacademics.in/journals/index.php/ijap

\section{INTRODUCTION}

Lornoxicam (LX) is a non-steroidal anti-inflammatory and painrelieving drug that is utilized for the treatment of joints inflammation, osteoarthritis, surgery, and sciatica [1]. LX inhibits both COX-1 and COX-2 and thus, has an inhibitory action on prostaglandin and thromboxane amalgamation [2]. However, LX is not preferred for oral administration for the following reasons. First, LX is classified according to the biopharmaceutical classification system (BCS) as a low solubility and high permeability drug (class II) $[3,4]$. Therefore, it suffers low oral bioavailability, along with short plasma half-life (3 to $5 \mathrm{~h}$ ) [5]. Second, its lipophilic nature and poor solubility in the acidic medium of the stomach cause local stomach irritation. Accordingly, topical delivery of LX will be advantageous, especially for patients that experience stomach problems $[6,7]$.

Nanosponges can be described as a colloidal structure based on hyper-crosslinked polymer comprising solid nanoparticles of nanosized cavities [8]. These colloidal nano-sized carriers have been proposed for drug delivery to solubilize lipophilic drugs and extend their release $[9,10]$. Moreover, nanosponges enhance the bioavailability of drugs and modify pharmacokinetic parameters [11, 12]. Furthermore, nanosponges are suitable means for delivering both lipophilic and hydrophilic substances due to their internal hydrophobic core and outer hydrophilic surface, offering excellent flexibility [13, 14]. Additionally, using hydrogel topical formulation as a delivery system can reduce irritation and improve retention on skin compared with other topical formulations $[15,16]$. It was reported that hydrogel increased drug skin absorption and permeation 10 times higher than oil-based formulations [17]. Moreover, hydrogel unique property (porosity) provides beneficial sustained and controlled drug delivery of hydrophobic drug via suitable release mechanism [18].

The study aimed to design a topical delivery system of LX nanosponges hydrogels to promote the skin permeation of LX and consequently enhance treatment efficacy. For this purpose, lornoxicam nanosponges were prepared using ethyl cellulose as a polymer by emulsion solvent evaporation method. The developed nanosponges were then incorporated into a hydrogel. The formulations were evaluated for in vitro drug release. Finally, nanosponge hydrogel was evaluated using the method of carrageenan-induced rat hind paw edema to assess the potential of anti-inflammatory activity of LX nanosponge gel.

\section{MATERIALS AND METHODS}

\section{Materials}

Lornoxicam was obtained as a gift from Hikma Pharmaceuticals Company (Egypt). Ethylcellulose (EC) (454.5 g/mol) and polyvinyl alcohol (PVA) of average molecular weight 40,000, were purchased from Sigma Aldrich (St. Louis, USA). Dichloromethane (DCM) was obtained from Algomhuria Medicine Trade Co. (Cairo, Egypt). Ethanol was acquired from ADWIC, (Egypt). Carboxyl methylcellulose sodium (CMC-Na) was kindly provided by EIPICO, (Egypt).

\section{Formulation of LX-loaded nanosponges}

Formulation of LX-loaded nanosponges was based on emulsion solvent evaporation [19]. Various amounts of ethyl cellulose and polyvinyl alcohol were utilized to prepare nanosponges (LS1-LS4). Dispersed phase was consisted of LX and EC and dissolved in $25 \mathrm{ml}$ of dichloromethane. It was added in portions to $150 \mathrm{ml}$ of an aqueous continuous phase containing a definite quantity of PVA. At that point, the blend was agitated at $1000 \mathrm{rpm}$ for $2 \mathrm{~h}$ on an overhead stirrer (VELP, DLS stirrer, Italy). The formed nanosponges were exposed to filtration using Whatman ${ }^{\circledR}$ membrane filters PTFE pore size $0.5 \mu \mathrm{m}$ ) and dried in a hot oven for $2 \mathrm{~h}$ at $40^{\circ} \mathrm{C}$ to remove any residual solvent. Nansponges were then kept in a vacuum desiccator. Table 1 shows the composition of LX loaded nanosponges formulations.

\section{Percentage entrapment efficiency (PEE)}

In a stoppered tube, nanosponges equivalent to $10 \mathrm{mg}$ lornoxicam were taken and the drug was extracted with $50 \mathrm{ml}$ of phosphate buffer solution (PBS) pH 6.8. The extracts were filtered using (Whatman® membrane filters PTFE pore size $0.5 \mu \mathrm{m}$ ) and transferred to $100 \mathrm{ml}$ of a volumetric flask and the volume was completed with PBS pH 6.8 [20]. The solutions were subjected to further dilution with the buffer and measured spectrophotometrically at $378 \mathrm{~nm}$. 
A calibration curve was plotted for lornoxicam in PBS (pH 6.8) in the range of $0.005-0.03 \mathrm{mg} / \mathrm{ml}$ at $378 \mathrm{~nm}$. A good linear relationship was observed between the concentration of lornoxicam and its absorbance $\left(\mathrm{R}^{2}=0.998\right)$. The specificity of the analytical method was performed using a blank formula which was prepared from all the used excipients except drug to check if any component of the formulation or the dissolution medium could interfere with the absorbance of lornoxicam at the selected wavelength.

Percentage entrapment efficiency was calculated by the following equation. PEE values given are the averages of three estimations.

$$
\mathrm{PEE}=\frac{\text { loading drug in nanosponges }}{\text { theoretical drug content }} \times 100
$$

Table 1: Formulation composition of lornoxicam-loaded nanosponges

\begin{tabular}{|c|c|c|c|c|}
\hline Formulation & LS1 & LS2 & LS3 & LS4 \\
\hline Drug: EC: PVA & $0.5: 1: 1$ & $0.5: 1: 2$ & $0.5: 1: 3$ & $0.5: 2: 2$ \\
\hline LX & $500 \mathrm{mg}$ & $500 \mathrm{mg}$ & $500 \mathrm{mg}$ & $500 \mathrm{mg}$ \\
\hline EC(g) & $1 \mathrm{~g}$ & $1 \mathrm{~g}$ & $1 \mathrm{~g}$ & $2 \mathrm{~g}$ \\
\hline PVA (g) & $1 \mathrm{~g}$ & $2 \mathrm{~g}$ & $3 \mathrm{~g}$ & $2 \mathrm{~g}$ \\
\hline Dichloromethane (DCM) $(\mathrm{ml})$ & $25 \mathrm{ml}$ & $25 \mathrm{ml}$ & $25 \mathrm{ml}$ & $25 \mathrm{ml}$ \\
\hline Dist. Water (ml) & $150 \mathrm{ml}$ & $150 \mathrm{ml}$ & $150 \mathrm{ml}$ & $150 \mathrm{ml}$ \\
\hline
\end{tabular}

\section{Particle size estimation of nanosponges}

Particle size of the dispersions was estimated utilizing a Zeta-sizer 3000 PCS (Malvern Instr., England) outfitted with a $5 \mathrm{~mW}$ heliumneon optical device. Estimations were made at $25^{\circ} \mathrm{C}$, edge $90^{\circ}$, run time in any event $180 \mathrm{~s}$. The samples were appropriately dispersed in deionized water preceding the estimations. The particle size values given are the averages of 3 estimations over 5 min each.

\section{Surface morphology}

Scanning electron microscopy (Qunta FEG 250, FEI, USA) was utilized to analyze surface morphology working at $20 \mathrm{kV}$. Lornoxicam nanosponges were kept on carbon sticky tape and vacuum dried. SEM photographs were recorded at magnification of 250X, 2000X, 5000X, 10000X.

\section{In vitro release of $\mathrm{LX}$ from nanosponges}

The in vitro release of $\mathrm{LX}$ from various nanosponges was performed utilizing the dialysis sac technique [21]. A sample equivalent to $1 \mathrm{mg}$ of $\mathrm{LX}$ was put in the regenerated cellulose dialysis sac (Mw cut-off at 12-14000 Da, Visking ${ }^{\circledR}$ dialysis tubing, UK) and both ends of the sac were firmly closed. The sac was immersed into a beaker containing
$150 \mathrm{ml}$ PBS of $\mathrm{pH} 6.8$ that served as the receptor cell. The beaker was placed in a shaker water bath at $37 \pm 0.5^{\circ} \mathrm{C}$ and agitated at 50 $\mathrm{rpm}$. Samples were compared to a solution of lornoxicam $(1 \mathrm{mg} / 5$ $\mathrm{ml}$ of PBS of pH 6.8; (LS0). For each sample, $3 \mathrm{ml}$ was withdrawn from the receptor cell at $1,2,3,4,5$ and $6 \mathrm{~h}$ and replaced by equivalent volumes of fresh release medium and kept up at a similar temperature. Drug concentrations were estimated spectrophotometrically at $\lambda_{\max } 378 \mathrm{~nm}$ [22] against equivalent PBS as a blank using Jenway spectrophotometer (Model 6105UV/Vis, England). The amounts of drug released were calculated based on the calibration curve made. Samples were tested in triplicate, and the average concentration was adopted.

\section{Formulation of LX nanosponges loaded hydrogels}

Hydrogels were prepared by adding CMC-Na to water and stirring with a mechanical stirrer at approximately $600 \mathrm{rpm}$ for $2 \mathrm{~h}$. Different hydrogels were formulated as illustrated in table 2 . The prepared dispersion was allowed to stand for $15 \mathrm{~min}$ to remove entrained air. At this point, LX nanosponges, propylene glycol and methanol, were added. Propyl and methylparaben were then added to the preparation as a preservative, and the volume was completed with water.

Table 2: Formulation design of lornoxicam-loaded nanosponges hydrogels

\begin{tabular}{lllll}
\hline Ingredients & \multicolumn{2}{l}{ Quantities \% (W/W) } & & G3 \\
\cline { 2 - 5 } & G0 & G1 & - & - \\
\hline LX powder & 0.5 & 5 & 5 & 5 \\
LX-loaded nanosponge (LS1) & - & 5 & 40 & 40 \\
Propylene glycol & 40 & 8 & 8 & 8 \\
Methanol & 8 & 1 & 1.5 & 2 \\
CMC-Na & 1 & 0.02 & 0.02 & 0.02 \\
Propyl paraben & 0.02 & 0.18 & 0.18 & 0.18 \\
Methyl paraben & 0.18 & 100 & 100 & 100 \\
Double distilled water (q. s.) & 100 & & \\
\hline
\end{tabular}

\section{Physicochemical evaluation of LX-loaded nanosponge hydrogels}

Determination of $\mathrm{pH}$ of LX-loaded nanosponges hydrogels

A digital pH meter (Model 420, ORION, USA) was used and calibrated utilizing standard buffers of $\mathrm{pH}$ of 4.0 and 7.0 before use. The glass electrode was immersed into the hydrogel and the $\mathrm{pH}$ readings were recorded at $25^{\circ} \mathrm{C}$ [23].

\section{Drug content estimation of LX-loaded nanosponge hydrogels}

One gram of LX nanosponge loaded hydrogels was blended with 100 $\mathrm{ml}$ of PBS pH 6.8: methanol (50:50) (v/v) and sonicated for $10 \mathrm{~min}$ to acquire a transparent solution [24]. LX concentrations were measured spectrophotometrically at $\lambda_{\max } 378 \mathrm{~nm}$. The percent of drug content was estimated in triplicate for each formulation.

\section{Viscosity measurement of LX-loaded nanosponge hydrogels}

The viscosity of the fabricated gel bases was assessed using a viscometer. The rotation of the spindle was at $10 \mathrm{rpm}$. Anton Paar MCR502,
SN81750818, and measuring cell: P-PTD200/TG, SN81720491, and measuring system: CP50-1/TG, SN31451 was used to measure the consistency of the fabricated gel bases. Samples were permitted to settle at room temperature for more than $30 \mathrm{~min}$ before estimation.

\section{In vitro release of LX from LX-loaded nanosponge hydrogels}

The in vitro release behavior of LX-loaded nanosponge hydrogels were investigated as prescribed previously compared to plain LX hydrogel.

\section{Skin irritation test}

The Draize test was performed to evaluate the irritation effect of developed LX-loaded nanosponge hydrogel formulation (G1) according to a previously published method. Animal ethical clearance certificate No. (181) was acquired by the Animal Ethics Committee from the Faculty of Pharmacy (Girl branch), Al-Azhar University, Cairo, Egypt, following recommendations for adequate care and use of laboratory pets (NIH publication No. 85-23, revised 1985). Albino rats ( $n=6 /$ group) were obtained from the animal 
house of Faculty of Pharmacy (Girl branch), Al-Azhar University, Cairo, Egypt. Rats were acclimated for seven days prior to the experiment and maintained on food and water. Their backs were shaved a day before the experiment. Gel formulation ( $0.5 \mathrm{~g})$ was applied on $4 \mathrm{~cm}^{2}$ of the hairless skin. Any changes on the skin were observed and recorded for 24,48 and $72 \mathrm{~h}$ after the hydrogel application. Formalin was used as positive control and plain ge formulation was used as a negative control. The degree of erythema was graded based on the original scale of Draize test [15].

\section{Anti-inflammatory efficacy}

Anti-inflammatory efficacy of formulated hydrogel was assessed utilizing the technique of carrageenan-induced rat hind paw edema $[25,26]$. Animal ethical clearance certificate No. (181) was acquired by the Animal Ethics Committee from the Faculty of Pharmacy (Girl branch), Al-Azhar University, Cairo, Egypt, following recommendations for adequate care and use of laboratory pets (NIH publication No. 85-23, revised 1985). Adult male albino rats (120$150 \mathrm{~g}$ ) were obtained from the animal house of Faculty of Pharmacy (Girl branch), Al-Azhar University, Cairo, Egypt. Rats were randomly divided into 2 groups $(n=6)$, acclimated for seven days prior to the experiment and maintained on food and water. Group 1 served as control and was treated with non-medicated plain gel. Group 2 and 3 were treated with plain LX hydrogel (G0), and LX-loaded nanosponge hydrogel (G1), respectively.

Throughout the experiment, LX-loaded nanosponge hydrogels and plain LX hydrogel $(0.5 \mathrm{~g})$ containing $5 \mathrm{mg}$ of LX were applied to the plantar surface of the left hind paw by delicately rubbing 5 times with the index finger. Bandages occluded the application region and remained in position for $2 \mathrm{~h}$. The bandages were separated, and the remaining gel was removed.

In rats, acute inflammation (paw edema) was caused by the injection of $0.1 \mathrm{ml}$ of $1 \%$ carrageenan solution in normal saline subcutaneously in the left hind paw sub-plant area and measured using a caliper device (micrometer). The injected paw thickness was evaluated immediately before carrageenan injection and at 1, 2, 3, 4, 5 and $6 \mathrm{~h}$ after carrageenan injection (MandW. Ltd, Sheffild, England). The edema inhibition was calculated as a percent from edema thickness of the control group [25].

\section{Statistical analysis}

The outcomes were analyzed statistically by means of one-way variance analysis (ANOVA), using GraphPad Prism version 8.1.2 software to determine the significance of differences between groups; a P value less than 0.05 was regarded as statistically significant.

\section{RESULTS}

\section{Percentage entrapment efficiency (PEE)}

Percentage entrapment efficiency has been established to ensure that an efficient amount of LX was entrapped in the nanosponges. Nanosponges had PEE ranged from $95.04 \% \pm 5.14$ to $99.32 \% \pm 2.25$ of LX. The results of PEE, Z-average, and PDI are shown in table 3.

Table 3: Percentage entrapment efficiency, Z-average, and PDI of measured nanosponges (values are mean \pm standard deviation of $n=3$ )

\begin{tabular}{|c|c|c|c|c|}
\hline Formulae & LS1 & LS2 & LS3 & LS4 \\
\hline LX: EC: PVA & $0.5: 1: 1$ & $0.5: 1: 2$ & $0.5: 1: 3$ & $0.5: 2: 2$ \\
\hline Percent Entrapment efficiency (PEE) & $98.87 \pm 4.25^{\mathrm{a}}$ & $95.04 \pm 5.14^{\mathrm{a}}$ & $98.97 \pm 3.87^{a}$ & $99.32 \pm 2.25^{\mathrm{a}}$ \\
\hline Z-average $(\mathrm{nm})$ & $545.5 \pm 1.19^{a}$ & $673.9 \pm 2.54^{b}$ & $818.7 \pm 1.66^{b}$ & $771.5 \pm 2.15^{c}$ \\
\hline Polydispersity index (PDI) & $0.320 \pm 0.05^{\mathrm{a}}$ & $0.654 \pm 0.05^{b}$ & $0.623 \pm 0.03^{b}$ & $0.468 \pm 0.06^{c}$ \\
\hline
\end{tabular}

Means (within the same row) with different superscript letters are statistically significant at $p>0.05$. Means (within the same row) with same superscript letters are statistically non-significant at $p>0.05$.

\section{Particle size estimation of nanosponges}

Particle size measurement was conducted to ensure that particles of the nanosponges are of the nanometer range. It was observed that all prepared nanosponges were in the nano-sized range (average particle size values ranged from $545.5 \pm 1.19 \mathrm{~nm}$ to $818.7 \pm 1.66 \mathrm{~nm}$ ), with a polydispersity index of $<1$ as shown in table 3 . The increase of PVA proportion brought about a significant increase in particle size at $p<0.001$ (table 3 ). The increase of EC: drug ratio by 4 folds led to a significant increase of particle size at $\mathrm{p}<0.001$.
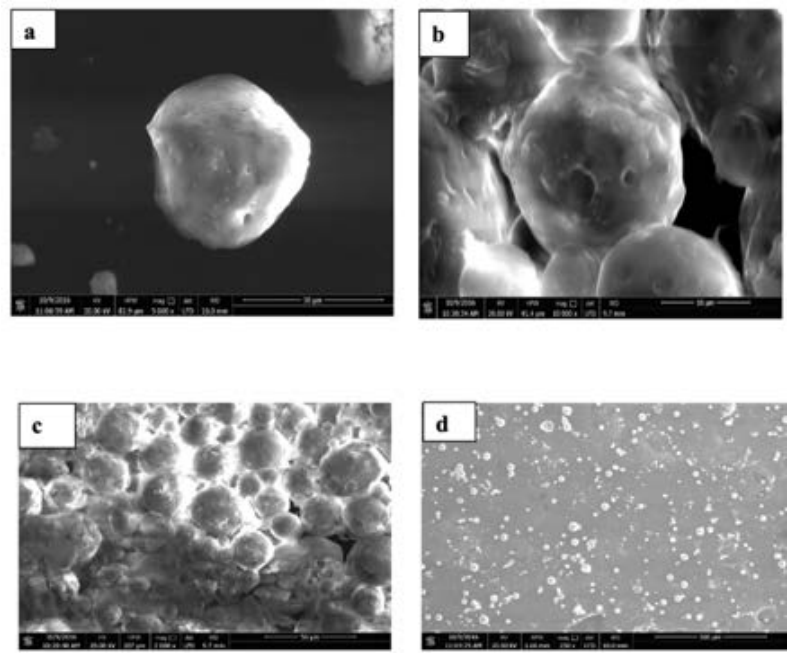

Fig. 1: SEM micrographs of nanosponges (LS4); (a) Magnification value (MV):5000x, (b) MV: 10000x, MV: (c) 2000x and (d) MV: 250x
One-way ANOVA results showed that the EC: PVA ratio had a significant impact on the average particle size (Z-average (d. nm)) of nanosponges $(\mathrm{p}<0.001)$.

\section{Surface morphology}

The nanosponge morphology was analyzed by scanning Quanta FEG 250 (FEI, USA) electron microscope (SEM). The SEM micrographs gave an idea regarding the morphological structure of nanosponges showing a round spongy structure with a smooth surface and fine holes (fig. 1).

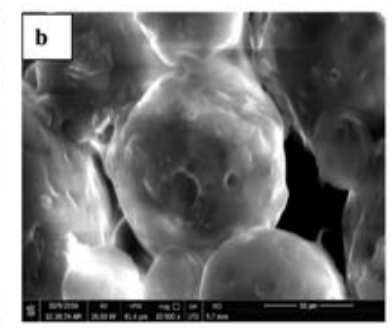

\footnotetext{
C.1:SEM micrographs of nanosponges (LS4); (a) Magnificaton vale (MV):5000x, (b) MV: 10000x, MV: (c) 2000xand (d) MV:250x
} 
In vitro release of $\mathrm{LX}$ from nanosponges and the kinetic analysis of the release data

Lornoxicam's in vitro release profile from formulated nanosponges was conducted in phosphate buffer solution (PBS) (pH 6.8) using the dialysis sac method compared to LX solution as a control (fig. 2). Lornoxicam's release from its solution was slow and only $38.4 \% \pm 1.9$ was released after $6 \mathrm{~h}$.

Nanosponge formulations improved LX release. Nanosponges (LS4, LS2 LS3, and LS1), has released about $(52.09 \pm 5.1,43.37 \pm 6.4,45.13 \pm 5.7$ and
$65.4 \pm 3.4) \%$, respectively of LX during $6 \mathrm{~h}$. Nonosponges were found to enhance the release by several folds [19]. Increasing PVA concentration significantly reduced the amount of $\mathrm{LX}$ released after $6 \mathrm{~h}$ at $\mathrm{p}<0.01$ [27]. LS3, with the largest particle size of $818.7 \pm 1.66 \mathrm{~nm}$, showed the least amount of drug release, while LS1 showed the highest release after $6 \mathrm{~h}$, so it was selected for further study.

It was observed that the in vitro release model best fitted to HixonCrowell release kinetic as their (r) value gave a higher value and ranges between 0.9929-0.9980. The Korsmeyer-Peppas release exponent (n) ranged between 0.67-0.79.

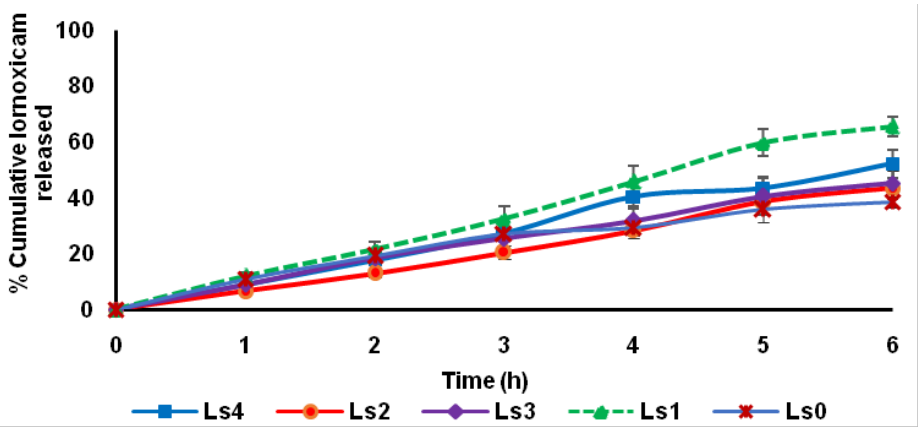

Fig. 2: In vitro release profiles of LX from LX-loaded nanosponges in comparison with control Ls0 (LX solution $1 \mathrm{mg} / \mathrm{ml} \mathrm{PBS} \mathrm{pH} \mathrm{6.8)} \mathrm{in} \mathrm{PBS}$ (pH 6.8) at $37^{\circ} \mathrm{C} \pm 0.5^{\circ} \mathrm{C}$. The values are the mean \pm standard deviation of $\mathrm{n}=3$. Abbreviations: Ls, formulations

\section{Physicochemical evaluation of LX nanosponge loaded hydrogels}

The $\mathrm{pH}$ values of LX-loaded nanosponges hydrogels were ranged between 7-8.1. LX content in the prepared hydrogels of nanosponges ranged between $85.9 \%-93.12 \%$. Fig. 3 illustrates the viscosity measurement of LX-loaded nanosponges hydrogels. The samples of G1,
G2 and G3 (detailed composition is presented in table 2) were found to be non-Newtonian liquids with shear-thinning properties. The viscosity of formulated hydrogels decreased with increasing the shear rate, as shown in fig. 3. It was found that G3 (CMC-Na 2\%), has a higher viscosity than other formulations, as indicated by the yield stress values. The yield stress values in Pa for G3, G2 and G1 were 105, 38 and 8, respectively.
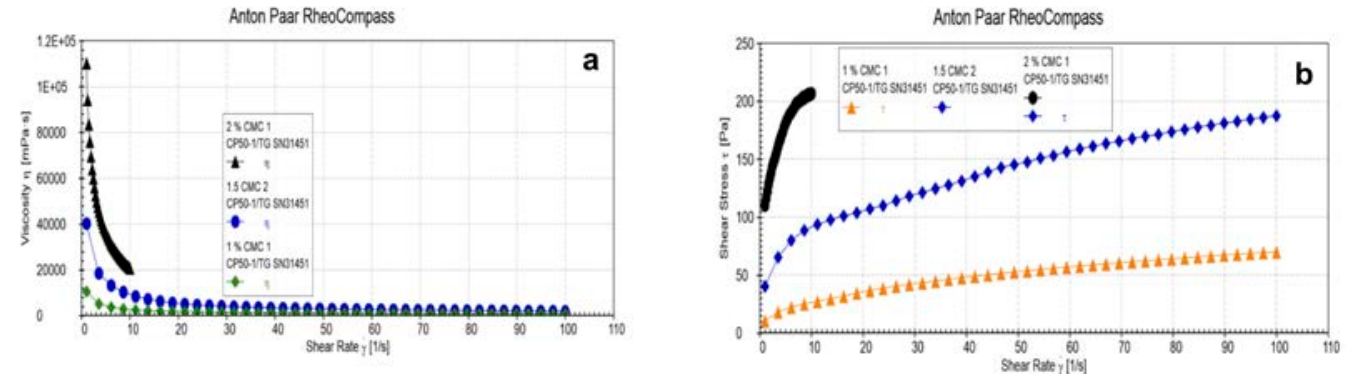

Fig. 3: Viscosity measurement; (a) Shear rate effect on the viscosity of various gel formulations and (b) Shear rate effect on the shear stress of various gel formulations

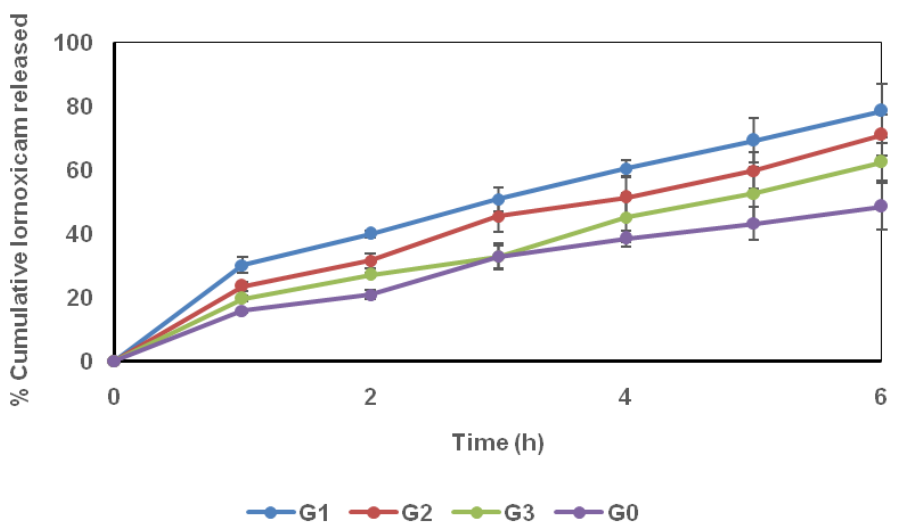

Fig. 4: In vitro release profiles of LX from topical hydrogels integrating LX-loaded nanosponges in phosphate buffer solution (pH 6.8) at 37 ${ }^{\circ} \mathrm{C} \pm 0.5{ }^{\circ} \mathrm{C}$. The values are the mean \pm standard deviation of $\mathrm{n}=3$. Abbreviations: $\mathrm{G}$, gel formulations 


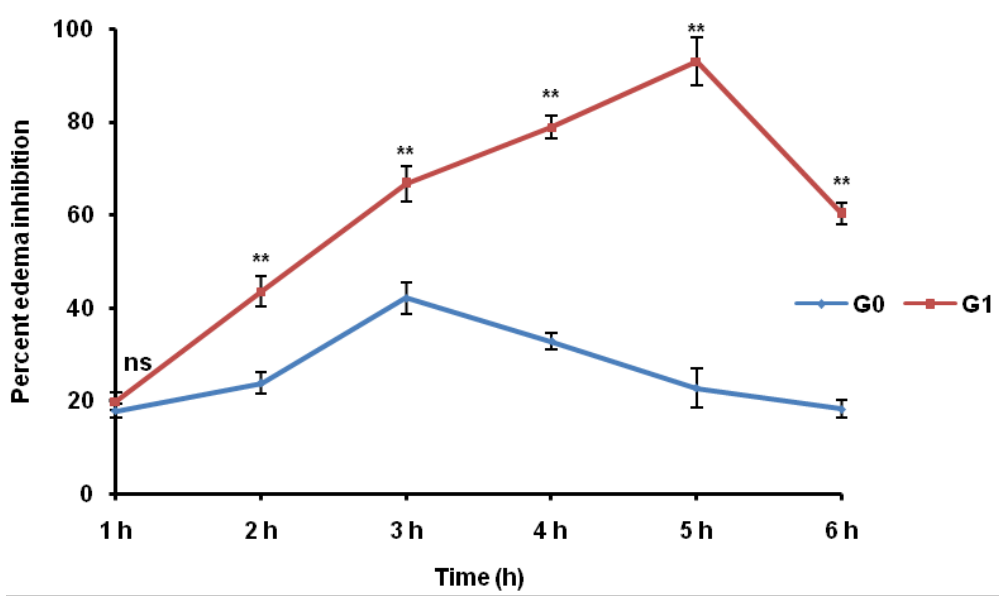

Fig. 5: Comparative percent edema inhibition of lornoxicam gel with lornoxicam nanosponge gel. The values are expressed as mean \pm standard deviation $(n=6)$. Abbreviations: G: Gel formulations, ns: values are not significant statistically compared to the control group where $\mathrm{P}>0.05$, ** Values are significantly different compared to control group where $\mathrm{P}<0.001$

\section{In vitro release of LX from LX-loaded nanosponges hydrogels}

Fig. 4 shows the in vitro release data of LX from various nanogels compared to the control LX gel (G0). It was found that G1 formulation released $39.81 \% \pm 1.11,60.41 \% \pm 2.5$ and $78.52 \% \pm 8.4$ after 2,4 and $6 \mathrm{~h}$, respectively. Furthermore, it was observed that amount of $\mathrm{LX}$ releases from G2 and G3 was lower than that released from G1.

Statistically, G1 showed a higher percentage of LX released compared to other gels at $\mathrm{p}<0.001$, after 2 and $4 \mathrm{~h}$. It can be observed that the release of formulations (G1, G2, and G3) exhibited Fickian transport according to Korsmeyer-Peppa's release model, which means that release always associated with diffusion mechanism while G0 exhibited non-Fickian transport.

\section{Skin irritation}

The mean erythemal score of the tested formulation G1 was 0.00 which indicated G1 was non-irritant to the skin as revelead by absence of erythema on the shaved skin.

\section{Anti-inflammatory activity}

Fig. 5 illustrates the anti-inflammatory activity LX-loaded nanosponge hydrogel (G1) compared plain LX gel (G0). Lornoxicamloaded nanosponge hydrogel (G1) produced \% edema inhibition $(66.63 \%)$ after $3 \mathrm{~h}$ while G0 produced only (42.11\%). Maximum \% edema inhibition of G1 was observed after $5 \mathrm{~h}(92.91 \%)$. After $6 \mathrm{~h}$, G0 showed \% edema inhibition (18.21\%) while \% edema inhibition of G1 was (60.15\%). Percent edema inhibition of G1 was significantly higher at $\mathrm{p}>0.001$ as compared to G0 at 2, 3, 4, 5 and 6 h. Moreover, it can be observed that the anti-inflammatory effects of G0 and G1 are correlated to the in vitro release results. For example, G1 showed LX release of $50.78 \%$ and generated $66.63 \%$ edema inhibition while G0 released $32.82 \%$ of LX and generated only $42.11 \%$ edema inhibition at $3 \mathrm{~h}$. Furthermore, G1 produced the maximum edema inhibition at $5 \mathrm{~h}$ (92.91\%), which in correlation with $69.25 \%$ of LX released.

\section{DISCUSSION}

The main scope of this study was to develop a new topical delivery system of LX using nansopnges as colloidal carriers suitable for such delivery. The colloidal properties of developed nanosponge formulations were investigated. Percentage entrapment efficiency values ensured that an efficient amount of LX was entrapped in the nanosponges. Particle size estimation confirmed that prepared nanosponges were in the nano-sized range with a polydispersity index of $<1$. The mean particle size of nanosponges was directly influenced by the drug: EC proportion. This may be attributed to the higher drug content and the lower amount of polymer available per nanosponge for encapsulation. Consequently, the thickness of the polymer wall is reduced and nanosponges are smaller [27]. The increase of EC ratio by 4 folds in LS4 led to a significant increase of particle size at $\mathrm{p}<0.001$ and this can be explained by the thick polymer arrangement caused by the polymer proportion increase. The high viscosity prevents the breaking of the emulsion into smaller droplets. Therefore, nanosponges with larger particle size are formed $[28,29]$. On the other hand, the low concentration of the EC increases the diffusion of dichloromethane (internal phase) into the aqueous solution (external phase), decreasing time needed to form droplets and thus reducing the particle size [13]. Moreover, increased particle size with increasing PVA proportion may be attributed to frothing, which leads to aggregates formation [15]. SEM micrographs revealed that the prepared nanosponges had a uniform round shape with a spongy structure and smooth surface. The fine holes present on nanosponge surface could be related to the diffusion of dichloromethane from the surface of the nanoparticles during the preparation phase $[29,30]$. In vitro release showed that LX's release from its solution was slow after $6 \mathrm{~h}$ and this may be attributed to its low solubility [31]. Nanosponge formulations improved LX release by several folds and this could be attributed to the disruption and diffusion of LX from the external surface of nanosponges at initial stage followed by a slow and sustained release of LX during $6 \mathrm{~h}$ [32]. Increasing PVA concentration significantly reduced the amount of LX released [27]. Such decrease could be explained by an increase in the thickness of the nanosponges' matrix as a result of increasing polymer concentration [33]. Increased thickness of nanosponge wall resulted in an extended diffusional way and thus, decreased LX release rates [13]. LS1 showed higher release after $6 \mathrm{~h}$ due to having the smallest particle size $(545.5 \pm 1.19 \mathrm{~nm})$, which was likely provided a large surface area for drug release, so it was selected for further study. It was noticed that the dissolution rate of nanosponges is extraordinarily affected by their surface area, porosity and particle size distribution [33]. Additionally, the drug release may be affected by the size of nansopnge holes that carry drug molecules [34]. The in vitro release kinetic data indicated anomalous non-fickian diffusion suggesting that release was controlled by a combination of diffusion and polymer relaxation. These results clarified that the drug release was controlled by the rate of solvent penetration into a nonswellable water-insoluble polymer such as ethylcellulose which controls drug release through the micropores present in their framework structure [35].

The physicochemical evaluation of LX nanosponge loaded hydrogels showed that hydrogels possessed both acceptable drug content and $\mathrm{pH}$ for topical application. Sample consistency is a significant parameter for topical formulations as it has to be applied in thin layers to the skin. It is, therefore beneficial to formulate a nonNewtonian flow system due to its low flow resistance when used under elevated shear circumstances [29]. The hydrogel samples were found to be non-Newtonian and shear-thinning liquids. It 
means that the viscosity decreased by increasing the shear rate. G3 (CMC-Na 2\%), showed a higher viscosity than other hydrogels indicating lower spreadability.

Developed nanogels revealed an enhanced release pattern compared to nanosponge formulations. This may be attributed to the penetration enhancement ability of propylene glycol incorporated in the nanogel formulations [36]. Moreover, it was found that G1 formulation released higher LX amount compared to G2 and G3 and this may be attributed to increased viscosity associated with increasing CMC-Na concentration as confirmed by the viscosity measurements [37]. Furthermore, the release pattern of developed nanogels exhibited Fickian transport according to KorsmeyerPeppa's release model, which means that release always associated with diffusion mechanism while G0 exhibited non-Fickian transport.

Furthermore, the Draize test revealed that the developed formulation was non-irritant to the skin, indicating its safe application. Anti-inflammatory assessment is based on the formulation's efficacy to inhibit the edema generated in hind paw after Carrageenan treatment. Maximum \% edema inhibition of developed nanogel formulation was observed after $5 \mathrm{~h}$ which indicate controlled and sustained anti-inflammatory response. After $6 \mathrm{~h}$, plain LX gel (control group) showed low \% edema inhibition compared to developed formulation. Developed nanogel produced significant enhancement anti-inflammatory activity compared to the control group for $6 \mathrm{~h}$. Moreover, it can be observed that the antiinflammatory effects of G0 and G1 are correlated to the in vitro release results. For example, G1 showed a LX release of $50.78 \%$ and generated $66.63 \%$ edema inhibition at $3 \mathrm{~h}$. G0 released $32.82 \%$ of LX and generated only $42.11 \%$ edema inhibition at the same time. Furthermore, G1 produced the maximum edema inhibition at $5 \mathrm{~h}$ (92.91\%) in correlation with $69.25 \%$ of LX released achieving a controlled anti-inflammatory response [38]. The findings support that nanosponges improved skin permeation and consequently enhanced the anti-inflammatory response [39].

\section{CONCLUSION}

Lornoxicam was efficiently encapsulated in EC nanosponge (drug: EC: PVA 0.5:1:1) using the emulsion solvent evaporation technique, followed by its incorporation into CMC-Na hydrogel. Formulated nanosponges possessed appropriate particle size with a sponge-like structure that was preserved within the colloidal gel. Nanospongebased hydrogels improved and controlled LX release for $6 \mathrm{~h}$ compared to control. The developed formulation showed no irritation effect on rat skin. Furthermore, formulated nanosponges demonstrated in vivo anti-inflammatory response that reached its maximum in $5 \mathrm{~h}$ indicating improved and controlled effect of the topical application for six hours. It could be inferred that EC based nanosponges would be an efficient nanocarrier for the dermal delivery of LX.

\section{FUNDING}

Nil

\section{AUTHORS CONTRIBUTIONS}

All the author have contributed equally.

\section{CONFLICTS OF INTERESTS}

The authors declare no conflict of interest.

\section{REFERENCES}

1. Helmy HS, El-Sahar AE, Sayed RH, Shamma RN, Salama AH, Elbaz EM. Therapeutic effects of lornoxicam-loaded nanomicellar formula in experimental models of rheumatoid arthritis. Int J Nanomed 2017;12:7015-23.

2. Zaid AN, Mousa A, Jaradat N, Bustami R. Lornoxicam immediate-release tablets: formulation and bioequivalence study in healthy mediterranean volunteers using a validated LC-MS/MS method. Clin Pharmacol Drug Dev 2017;6:564-9.

3. Gadade D, Kulkarni D, Rathi P, Pekamwar S, Joshi S. Solubility enhancement of lornoxicam by crystal engineering. Indian J Pharm Sci 2017;79:277-86.

4. Nijhawan M, Santhosh A, Babu PR, Subrahmanyam CV. Solid state manipulation of lornoxicam for cocrystals-physico- chemical characterization. Drug Dev Industrial Pharm 2014;40:1163-72.

5. Marzouk M, Ammar A, Darwish M, Ali H. Preformulation, development and in vitro study of bilayer tablets of lornoxicam. Asian J Pharm Health Sci 2017;7:1769-79.

6. Dasgupta S, Ghosh SK, Ray S, Kaurav SS, Mazumder B. In vitro and in vivo studies on lornoxicam loaded nanoemulsion gels for topical application. Curr Drug Delivery 2014;11:132-8.

7. Yadav SK, Mishra MK, Tiwari A, Shukla A. EMULGEL: a new approach for enhanced topical drug delivery. Int J Curr Pharm Res 2016;9:15-9.

8. Tejashri G, Amrita B, Darshana J. Cyclodextrin based nanosponges for pharmaceutical use: a review. Acta Pharm (Zagreb, Croatia) 2013;63:335-58.

9. Khalifa MKA, Salem HA, Shawky SM, Eassa HA, Elaidy AM. Enhancement of zaleplon oral bioavailability using optimized self-nano emulsifying drug delivery systems and its effect on sleep quality among a sample of psychiatric patients. Drug Delivery 2019;26:1243-53.

10. Shringirishi M, Prajapati SK, Mahor A, Alok S, Yadav P, Verma A. Nanosponges: a potential nanocarrier for novel drug delivery-a review. Asian Pac J Trop Dis 2014;4:S519-S26.

11. Trotta F, Dianzani C, Caldera F, Mognetti B, Cavalli R. The application of nanosponges to cancer drug delivery. Expert Opin Drug Delivery 2014;11:931-41.

12. Sujitha YS, Muzib YI. Formulation and optimization of quercetin loaded nanosponges topical gel: ex vivo, pharmacodynamic and pharmacokinetic studies. Int J Appl Pharm 2019;11:156-65.

13. Selvamuthukumar S, Anandam S, Krishnamoorthy K, Rajappan $\mathrm{M}$. Nanosponges: a novel class of drug delivery system-review. J Pharm Pharm Sci 2012;15:103-11.

14. Wadhwa A, Mathura V, Lewis SA. Emerging novel nanopharmaceuticals for drug delivery. Asian J Pharm Clin Res 2018;11:35-42.

15. Aldawsari HM, Badr Eldin SM, Labib GS, El-Kamel AH. Design and formulation of a topical hydrogel integrating lemongrassloaded nanosponges with an enhanced antifungal effect: in vitro/in vivo evaluation. Int J Nanomed 2015;10:893.

16. Ganesh R Pawbake, Satish V Shirolkar. Microemulgel: a promising approach to improve the therapeutic efficacy of drug. JCR 2020;7:1137-43.

17. Haltner Ukomadu E, Sacha M, Richter A, Hussein K. Hydrogel increases diclofenac skin permeation and absorption. Biopharm Drug Dispos 2019;40:217-24.

18. Narayanaswamy R, Torchilin VP. Hydrogels and their applications in targeted drug delivery. Molecules 2019;24:603.

19. Pushpalatha R, Selvamuthukumar S, Kilimozhi D. Cyclodextrin nanosponge based hydrogel for the transdermal co-delivery of curcumin and resveratrol: development, optimization, in vitro and ex vivo evaluation. J Drug Delivery Sci Technol 2019;52:55-64.

20. Panday P, Shukla N, Sisodiya D, Jain V, Mahajan SC. Design and characterization of microsponge loaded controlled release epicutaneous gel of lornoxicam. Appl Med Res 2015;1:16-21.

21. Bhardwaj U, Burgess DJ. Physicochemical properties of extruded and non-extruded liposomes containing the hydrophobic drug dexamethasone. Int J Pharm 2010;388:181-9.

22. Al-Suwayeh SA, Taha EI, Al-Qahtani FM, Ahmed MO, Badran MM. Evaluation of skin permeation and analgesic activity effects of carbopol lornoxicam topical gels containing penetration enhancer. Sci World J 2014. https://doi.org/10.1155/2014/127495.

23. Das B, Nayak AK, Nanda U. Topical gels of lidocaine $\mathrm{HCl}$ using cashew gum and carbopol 940: preparation and in vitro skin permeation. Int J Biol Macromol 2013;62:514-7.

24. Osmani RAM, Kulkarni PK, Shanmuganathan S, Hani U, Srivastava A, Prerana M, et al. A 32 full factorial design for development and characterization of a nanosponge-based intravaginal in situ gelling system for vulvovaginal candidiasis. RSC Adv 2016;6:18737-50.

25. Gao S, Tian B, Han J, Zhang J, Shi Y, Lv Q, et al. Enhanced transdermal delivery of lornoxicam by nanostructured lipid carrier gels modified with polyarginine peptide for treatment of carrageenan-induced rat paw edema. Int J Nanomed 2019;14:6135. 
26. Shaikh S, Joshi YM, Kadam V. Comparative study of antiinflammatory activity of aqueous and methanolic extracts of hibiscus cannabinus leaf (malvaceae). Int J Pharm Pharm Sci 2016;8:64-8.

27. Moin A, Deb TK, Osmani RAM, Bhosale RR, Hani U. Fabrication, characterization, and evaluation of the microsponge delivery system for facilitated fungal therapy. J Basic Clin Pharm 2016;7:39.

28. Pawar P, Gholap A, Kuchekar A, Chellam B, Mali A. Formulation and evaluation of optimized oxybenzone microsponge gel for topical delivery. J Drug Delivery 2015:1-9. Doi:10.1155/2015/261068.

29. Rajab NA, Jawad MS. Formulation and in vitro evaluation of piroxicam microsponge as a tablet. Int J Pharm Pharm Sci 2016;8:104-14.

30. Jilsha G, Viswanad V. Nanosponge loaded hydrogel of cephalexin for topical delivery. Int J Pharm Sci Res 2015;6:2781.

31. Anumolu PD, Sunitha G, Bindu SH, Satheshbabu PR, Subrahmanyam CVS. Development and validation of discriminating and biorelevant dissolution test for lornoxicam tablets. Indian J Pharm Sci 2015;77:312.
32. Neela S, Uppuluri KB. Formulation and in vitro evaluation of piroxicam loaded BSA nanospheres by desolvation. J Nanomed Nanotechnol 2015;6:1.

33. Sharma R, Pathak K. Polymeric nanosponges as an alternative carrier for improved retention of econazole nitrate onto the skin through topical hydrogel formulation. Pharm Dev Technol 2011;16:367-76.

34. Patel B, Bagade O, Ramteke K, Patel R, Awsarkar V. An assessment on preparations, characterization, and poles apart appliances of nanosponge. Int J PharmTech Res 2014;6:1898-907.

35. Bhandare CR, Katti SA. Formulation of microsponges of risperidone HCl. Int J Res Pharm Chem 2016;6:518-27.

36. Aithal PA, Mulla K, Shabaraya A. Formulation and evaluation of nanosponge based topical gel preparation of dapsone; 2019. DOI:10.13140/RG.2.2.36330.26562

37. Patel J, Patel B, Banwait H, Parmar K, Patel M. Formulation and evaluation of topical aceclofenac gel using different gelling agent. Int J Drug Dev Res 2011;3:156-64.

38. El-Assal MI. Nano-sponge novel drug delivery system as carrier of anti-hypertensive drug. Int J Pharm Pharm Sci 2019;11:47-63.

39. Abbas N, Parveen K, Hussain A, Latif S, Uz Zaman S, Shah PA, et al. Nanosponge-based hydrogel preparation of fluconazole for improved topical delivery. Trop J Pharm Res 2019;18:215-22. 